\title{
Rechtsprechung
}

\section{Rückabwicklung eines Kaufvertrages über eine im Wege einer öffentlichen Kunstauktion angebotene und erworbene Buddha-Statue}

Oberlandesgericht München, Endurteil vom 26. Juni 2012 - 5 U 2038/11

\author{
1. Der Umstand einer neuzeitlichen Fälschung stellt sich in rechtlicher Hinsicht als Sachmangel im Sinne der \\ anwendbaren BGB-Vorschriften zum Kaufrecht dar.
}

2. Da dieser Mangel objektiv nicht behebbar ist, war der Kläger gemäß $\S 437$ Nr. 2, 440, 323, 326 Abs. 5,275 Abs. 1 BGB ohne Nachfristsetzung zum Rücktritt berechtigt.

3. Ziff. 7 a) der als allgemeine Geschäftsbedingungen formulierten Versteigerungsbedingungen steht dem nicht entgegen, denn diese Klausel ist zum einen überraschend im Sinne von $\$ 305 c$ BGB und daher nicht Vertragsbestandteil geworden und zum anderen wegen unangemessener Benachteiligung der Vertragspartei des Verwenders gemäß § 307 BGB unwirksam. (Leitsätze der Redaktion)

I.

- Auf die Berufung des Klägers wird das Urteil des Landgerichts München I vom 06. April 2011, Az. 23 O 24119/10, unter Abweisung der weiterreichenden Berufung wie folgt abgeändert:

1. Der Beklagte wird verurteilt, an den Kläger $21.634,51 €$ nebst Zinsen in Höhe von 5\%-Punkten über dem Basiszinssatz aus 20.295,00€ seit 04. September 2010 sowie Zinsen in Höhe von 5\%-Punkten über dem Basiszinssatz aus 1.339,51€ seit 04. Januar 2011 zu zahlen Zug um Zug gegen Übereignung und Übergabe der als "Sitzender Buddha. Dhyana Asana" bezeichneten Statue [...].

2. Es wird festgestellt, dass sich der Beklagte im Annahmeverzug befindet.

3. Im Übrigen wird die Klage abgewiesen.

II.

Von den Kosten des Rechtsstreits tragen der Kläger 10\% und der Beklagte $90 \%$.

III.

Das Urteil ist vorläufig vollstreckbar. Die Parteien können die Vollstreckung durch Sicherheitsleistung in Höhe von $110 \%$ des vollstreckbaren Betrages abwenden, wenn nicht die jeweils vollstreckende Partei zuvor Sicherheit in Höhe von 110\% des zu vollstreckenden Betrages leistet.

IV.

Die Revision gegen dieses Urteil wird zugelassen.

\section{V.}

Der Streitwert des Berufungsverfahrens wird auf 23.889, $51 €$ festgesetzt.

\section{Gründe}

I.

Die Parteien streiten um Gewährleistungsansprüche aus einem Kaufvertrag über eine im Wege einer öffentlichen Kunstauktion angebotene und erworbene Buddha-Statue.

Der Beklagte, ein öffentlich bestellter und vereidigter Auktionator im Sinne von $\S 34$ b Abs. 5 GewO, betreibt als Einzelunternehmer in L. am [...] ein Auktionshaus, in dem er die Internationalen [...]-Kunstauktionen durchführt. Im Katalog zur 103. [...]-Kunstauktion, die vom 03. Dezember 2009 bis 05. Dezember 2009 stattfand, war unter der Losnummer 1131 eine vom Streitverkündeten Dr. P. eingelieferte Buddhaskulputur abgebildet, die folgendes Aussehen hat: [...]

Sie war wie folgt beschrieben:

"Sitzender Buddha. Dhyana Asana. Hände fehlen. Marmor mit Wurzelspuren. China, Sui-Dynastie, 581-618. H 40 $\mathrm{cm}$. Es handelt sich wahrscheinlich um den historischen Buddha S. Der regelmäßige Verlauf der ziemlich flachen Falten und das enge Anliegen des Gewandes am Körper entsprechen noch dem nördlichen Ch'i-Stil. Museall 3.800,00 €" [...].

Bei der am 04. Dezember 2009 auf der Grundlage der Versteigerungsbedingungen [...] durchgeführten Auktion wurde die Skulptur dem Kläger für 20.295,00 € incl. Aufgeld zugeschlagen (Rechnung [...]). Auf den Kaufpreis leistete der in Zü. 
wohnhafte Kläger am 04. Dezember 2009 eine Teilzahlung von $10.762,40 €$ und am 09. Dezember 2009 eine Schlusszahlung von $10.000,00 €$.

Unter Ziff. 7 der Versteigerungsbedingungen sind Gewährleistung und Haftung des Versteigerers wie folgt geregelt:

„a) Der Käufer kann gegen das Auktionshaus keine Einwendungen oder Ansprüche wegen Sachmängeln erheben. Das Auktionshaus wird jedoch begründete Mängelrügen, die ihm innerhalb einer Frist von einem Jahr seit Übergabe der Sache vom Käufer angezeigt werden, gegenüber dem Einlieferer geltend machen, wenn der Käufer die dafür notwendigen sachlichen und rechtlichen Voraussetzungen nachweist.

b) Die Haftung des Auktionshauses auf Schadensersatz für Vermögensschäden, gleich aus welchem Rechtsgrund, ist ausgeschlossen, es sei denn, dem Auktionshaus fiele Vorsatz oder grobe Fahrlässigkeit zur Last.

c) Objekte mit einem Ausrufpreis bis 100 Euro sind von Reklamationen wegen Sachmängeln ausgeschlossen."

Die "Grundlagen der Versteigerung" sind in Ziffer 2 der Versteigerungsbedingungen wie folgt formuliert:

„a) Die Versteigerung ist freiwillig und öffentlich iSd § 383 Abs. 3 BGB. Sie wird durch das Auktionshaus als Kommissionär im eigenen Namen für Rechnung der Einlieferer durchgeführt, die unbenannt bleiben.

b) Die zur Versteigerung kommenden Gegenstände können vor der Auktion besichtigt und geprüft werden. Die Sachen sind gebraucht. Sie werden in dem Zustand versteigert, in dem sie sich bei der Auktion befinden. Die Katalogangaben sind nach bestem Wissen und Gewissen vorgenommen, sie sind aber nicht Teil der vertraglich vereinbarten Beschaffenheit der Gegenstände; das gleiche gilt für deren Bezeichnung beim Ausruf. [...]"

Der Kläger behauptet unter Vorlage eines vorprozessual in Auftrag gegebenen Gutachtens des Dr. R. N. [...], Geschäftsführer der A.XXX GmbH, die erworbene Skulptur sei eine neuzeitliche Fälschung. Er begehrt deswegen mit der am 21. Dezember 2010 bei Gericht eingegangenen Klage die Rückabwicklung des Kaufvertrages sowie Ersatz des infolge der Wechselkursänderungen entstandenen Währungsschadens gegen Rückgabe der Statue. Nachdem seine vorprozessualen Bemühungen erfolglos geblieben sind und auch der Einlieferer Dr. P., dessen Identität der Beklagte dem Kläger offengelegt hat, eine Regulierung abgelehnt hat, verfolgt er diesen Anspruch mit der Klage, mit der er Zahlung von insgesamt 23.889,51 € (Kaufpreis: 20.295,00 $€$, Gutachterkosten: 1.339,51 €, Währungsschaden: 2.255,00 €) nebst Zinsen in Höhe des gesetzlichen Verzugszinssatzes seit dem 03. September 2010 aus dem Kaufpreis sowie den Gutachterkosten und aus dem überschießenden Betrag seit Rechts- hängigkeit Zug um Zug gegen Rückgabe der Statue, und daneben die Feststellung des Annahmeverzuges des Beklagten begehrt.

Dem ist der Beklagte entgegen getreten. Er bestreitet eine Fälschung und beruft sich im Übrigen auf die Versteigerungsbedingungen.

Am 06. April 2011 hat das Landgericht ein klageabweisendes Urteil erlassen. Infolge wirksam vereinbarten Gewährleistungsausschlusses stünden dem Kläger gegen den Beklagten keine Ansprüche zu, weshalb über den behaupteten Sachmangel kein Beweis zu erheben sei. Der Beklagte sei nicht gehindert, sich auf den vereinbarten Ausschluss zu berufen, weil er die ihm gegenüber dem Einlieferer obliegenden Sorgfaltspflichten bei der Hereinnahme des Kommissionsgutes nicht verletzt habe. Die Objektbeschreibung im Katalog sei auch nicht als Beschaffenheitsvereinbarung über die Kaufsache zu verstehen.

Gegen diese Entscheidung wendet sich der Kläger mit der Berufung, mit der er seinen Klageantrag unverändert weiter verfolgt. Der Beklagte hält das Ersturteil für richtig.

Der Senat hat am 22. Juli 2011 Hinweise erteilt [...] sowie Beweis erhoben über die Echtheit der Statue durch Einholung eines Gutachtens der Sachverständigen Dr. R., Kuratorin des Museums für Ostasiatische Kunst in Köln, und durch Vernehmung des Dr. N. als sachverständigen Zeugen. Wegen des Ergebnisses der Beweisaufnahme wird auf das schriftliche Sachverständigengutachten vom 20. Dezember 2011 [...] nebst Ergänzungsgutachten vom 28. März 2012 [...] und auf die Sitzungsniederschriften vom 24. April 2012 [...] und 25. Mai 2012 [...] verwiesen. Ergänzend wird auf die tatbestandlichen Feststellungen im landgerichtlichen Urteil Bezug genommen.

II.

Die Berufung hat hinsichtlich des Rückabwicklungsbegehrens Erfolg; hinsichtlich des daneben beanspruchten Ersatzes des Währungsschadens verbleibt es bei der Klageabweisung.

1. Gemäß dem hier (noch) anwendbaren Lugano-Übereinkommen (Übereinkommen über die gerichtliche Zuständigkeit und die Vollstreckung gerichtlicher Entscheidungen in Zivilund Handelssachen geschlossen in Lugano) vom 16. September 1988, nämlich Art. 2 Abs. 1 LugÜ, sind die Gerichte der Bundesrepublik Deutschland als desjenigen Staates, in dessen Hoheitsgebiet der Beklagte seinen Wohnsitz hat, für die Entscheidung des Rechtsstreits international zuständig.

2. Der Kläger ist wirksam vom Kaufvertrag zurückgetreten und kann daher gemäß §§ 346 Abs. 1, 433 Abs. 1 Satz 2, 434 Abs. 1 Satz 2 Nr. 1, 437 Nr. 2, 440, 323, 326 Abs. 5, 275 Abs. 1 BGB von seinem Vertragspartner, dem Beklagten, Rückgewähr der erbrachten Vertragsleistungen Zug um Zug gegen Rückgewähr der empfangenen Leistungen fordern. 
2.1. Bei der Kaufsache handelt es sich um eine neuzeitliche Fälschung. Dies steht in tatsächlicher Hinsicht aufgrund der durchgeführten Beweisaufnahme fest.

(1) Auf der Grundlage eines stilistischen Vergleiches hat die Sachverständige Dr. R., die infolge jahrzehntelanger intensiver Befassung mit ostasiatischer Kunst, insbesondere mit BuddhaFiguren, über eine überragende Fachkunde für die hier vorzunehmende Vergleichung verfügt, die streitgegenständliche Skulptur als Fälschung beurteilt.

Die Sachverständige befasste sich seit Beginn ihres Studiums vor 24 Jahren durchgehend mit ostasiatischer Kunst. Im Zentrum ihrer Magisterarbeit und ihrer gegenwärtig in Ausarbeitung befindlichen Habilitationsschrift stehen buddhistische Figuren. Die Sachverständige, die sich nach ihrem Bekunden seit 1998 mehrmals und teils für mehrere Monate in Nordchina aufgehalten und dort nahezu alle wesentlichen Tempel, zahlreiche Höhlentempel, zudem diverse Ausstellungen, Museen und Depots, in die die Ausgrabungsfunde eingeliefert werden, besucht hat, verfügt über eine reichhaltige Kenntnis über die stilistischen Eigenschaften und Merkmale von Buddha-Skulpturen aus der hier maßgeblichen Epoche, der Sui-Dynastie, gewonnen aus einer unmittelbaren eigenen Wahrnehmung verlässlicher Objekte vor Ort. Sie ist daher zur Durchführung der Begutachtung geeignet; ihre Beurteilung ist verlässlich.

Die Sachverständige hat ausgeführt, sie sei aufgrund der vorgefundenen Auffälligkeiten bei der streitgegenständlichen Skulptur davon überzeugt, dass es sich um eine Fälschung handele. Ihre Beurteilung stützt die Sachverständige auf drei Hauptargumente: Der Jade-Ring, ein Schmuckgegenstand, befindet sich auf der Rückseite des Gewandes; die Ausführung des Gewandes auf der Vorderseite passt nicht zur Ausführung auf der Rückseite; die Haarkalotte des Hinterkopfes weist einen ungewöhnlich hohen, kreisrunden Haaransatz auf.

Der Jade-Ring werde richtigerweise - wie es für einen Schmuckgegenstand auch zu erwarten sei - auf der Vorderseite des Gewandes getragen. Soweit der Beklagte auf die dem Sitzungsprotokoll vom 25. Mai 2012 als Bildtafel 3 beigeheftete Ablichtung einer weiteren Statue mit Jade-Ring auf der Gewandrückseite verweist, lässt sich daraus schon deshalb kein stichhaltiger Einwand gegen die Ausführungen der Sachverständigen herleiten, weil die Fotografie eine im Besitz des Einlieferers Dr. P. befindliche Statue ungesicherter Herkunft zeigt. Dem Antrag des Beklagten, die Frist zur Stellungnahme auf die Ausführungen der Sachverständigen zu verlängern, um die erwartete Antwort einer vom Streitverkündeten veranlassten Anfrage an das [...] Museum vom 29. Mai 2012 nachreichen zu können und so zu beweisen, dass auch eine im [...] Museum in [...] ausgestellte Buddha-Statue einen Jade-Ring auf der Gewandrückseite aufweise, war nicht stattzugeben. Ausweislich der Email-Anfrage des Streitverkündeten (Anlage BE 12 zum Schriftsatz des Beklagten vom 12. Juni 2012) hat dieser um die Überlassung einer Ablichtung von der Rückseite der dort be- zeichneten Statue (offenbar derjenigen Statue, deren vorderseitige Ansicht mit Anlage BE 6 vorgelegt worden ist) gebeten, um herauszufinden, ob sich auf der Rückseite ein Jadering befindet ( „It is expecially important for us to see, if there is a jade ring at the back of the figure holding the strings."; so auch in der Mail vom 09. Juni 2012: „Mal sehen, ob die Rückseite einen Jadering aufweist."). Die Beweisbehauptung, die bezeichnete Skulptur trage auf der Rückseite einen Jadering, stellt sich daher als eine ins Blaue hinein aufgestellte Behauptung ohne jede Tatsachengrundlage dar.

Tatsächlich will der Beklagte lediglich den Rechtsstreit verzögern und weitere Ermittlungen anstellen. Für die Richtigkeit seiner Behauptung gibt es nicht den geringsten Anhalt.

Dass die Ausführung des Gewandes auf der Rückseite nicht die Fortsetzung des vorderseitigen Gewandes darstellt, hat die Sachverständige anhand der vorliegenden Ganzkörper-Ablichtungen von Vor- und Rückseite der streitgegenständlichen Figur erläutert. Die völlig unbedeckte rechte Körperhälfte auf der Rückseite passt nicht zusammen mit der Darstellung der stofflichen Bekleidung auf der Vorderseite. Dass es sich hierbei um eine untypische Auffälligkeit handelt, weil die Skulpturen aus verlässlichen Funden eine in sich logische Darstellung des Gewandes zeigen, ist nachvollziehbar und überzeugend.

Soweit der Beklagte die Ausführungen der Sachverständigen dahingehend, dass die hohe runde Haarkalotte unüblich sei, in Zweifel zieht, kann dem gleichfalls nicht gefolgt werden. Die dem Sitzungsprotokoll vom 25. Mai 2012 als Bildtafel 1 beigefügte Fotografie mit vergleichbar hoher und runder Haarkalotte zeigt wiederum eine Figur aus der Sammlung des Streitverkündeten, die zudem - worauf die Sachverständige hingewiesen hat - untypische Einkerbungen auf der Rückseite aufweist, weshalb die Skulptur insgesamt als "hochproblematisch" anzusehen sei. Die Bildtafel 2 zum Sitzungsprotokoll vom 25. Mai 2012 wiederum zeigt gemäß den Erläuterungen der Sachverständigen eine völlig andere Figur, nämlich einen Boddhisattva, aus einem nicht vergleichbaren Zeitstil, weshalb die dortige Haartracht - ohnehin keine kreisrunde Haarkalotte - nichts für die Beurteilung der streitgegenständlichen Skulptur hergibt. Soweit der Beklagte auf die an das [...] Museum gerichtete Anfrage (siehe bereits oben) verweist, ist auch in diesem Zusammenhang dem Fristverlängerungsgesuch nicht stattzugeben. Dem Beklagten und dem Streitverkündeten ist die Rückseite der Figur ausweislich des Inhalts der Anfrage nicht bekannt. Dafür, dass die Figur einen vergleichbaren Haaransatz aufweisen könnte, gibt es keinerlei Hinweis.

Demgegenüber hat die Sachverständige eine Vielzahl verlässlicher Funde im Original besichtigt und sich damit wissenschaftlich befasst. Ihre Beobachtung, dass die hier wiedergegebenen Merkmale auffällig und ungewöhnlich sind, ist daher verlässlich, die daraus gezogene Schlussfolgerung, dass die Skulptur nicht aus der Sui-Dynastie stammt, sondern eine neuzeitliche Fälschung darstellt, überzeugend. Den Umstand, dass 
sich die Häufung stilistischer Fehler auf der Rückseite der Skulptur befindet, hat die Sachverständige überzeugend als weiteren Hinweis auf das Vorliegen einer Fälschung damit begründet, dass von Ausstellungsstücken in der Regel die Vorderseiten, nicht aber die Rückseiten abgelichtet werden, was bei der Gestaltung von Rückseiten durch Fälscher eine höhere Fehleranfälligkeit begründet.

Die weiteren stilistischen Ungereimtheiten - wie die unproportionale Betonung des Kopfes, die füllige Gestaltung des Faltenwurfes auf der Gewandrückseite im Gegensatz zu der stilisierten Darstellung auf der Vorderseite, die ungewöhnliche Verknotung der Kordel auf der Vorderseite - stellen weitere von der Sachverständigen aufgezeigte Auffälligkeiten dar, die den aus den Hauptgesichtspunkten gezogenen Schluss auf das Vorliegen einer modernen Fälschung stützen.

(2) Zu diesen Erkenntnissen aus einem fachkundigen, stilistischen Vergleich treten die Untersuchungsergebnisse des sachverständigen Zeugen Dr. N. hinzu. Dieser hat vor dem Senat seine bereits in der Anlage K 6 beschriebene Vorgehensweise nachvollziehbar erläutert.

Die von der Oberfläche der Skulptur genommenen Proben weisen nach den Feststellungen des Zeugen hinsichtlich der Konzentration von Kalzium- und Magnesiumkarbonat keinen Unterschied zu der aus dem Inneren des Objektes entnommenen Probe auf. Der Sachverständige hat überzeugend ausgeführt, dass diese Feststellung gegen die Annahme eines Alters von angeblich bereits 1400 Jahren spricht. Bei einer derart langen Lagerzeit in einer humiden Umgebung - eine solche sei Voraussetzung für eine Versinterung, wie sie dem Betrachter des streitgegenständlichen Objektes mit den Wurzelspuren vor Augen geführt werden solle - sei eine Abnahme des leichter wasserlöslichen Kalziumkarbonats und eine entsprechende Anreicherung von Magnesiumkarbonat an der Oberfläche zu erwarten.

Soweit der Beklagte meint, die Ausführungen des Sachverständigen zur angeblichen Verwitterung seien widersprüchlich, kann dem nicht gefolgt werden. Der Sachverständige hat überzeugend dargelegt, dass er auf der Grundlage seiner Materialuntersuchungen keine Verwitterung des Steins feststellen konnte. Die Beschreibung der Vorgehensweise auf Seite 2 des schriftlichen Gutachtens dahingehend, er habe kleine Proben „der verwitterten Oberfläche (03) und der Verkrustung (02 und 04; Sinter?) entnommen, sowie zum Vergleich einen Splitter des unverwitterten Materials (05; Basismaterial)“ beinhaltet nicht die Feststellung, dass die Oberfläche, von der die Materialprobe 03 genommen wurde, tatsächlich verwittert war - eine solche Verwitterung hat der Sachverständige ausweislich der festgehaltenen und vor dem Senat erläuterten Materialuntersuchung gerade nicht festgestellt. Mit der dortigen Darstellung der Vorgehensweise wird vielmehr lediglich der Grund für die Auswahl der Probenahmen bezeichnet, nämlich der Umstand, dass die Probe 03 aufgrund ihrer Entnahmestelle im Vergleich zur Probe 05 aufgrund deren Entnahmestelle unter der Voraussetzung einer entsprechend langen Lagerdauer eine typische Materialunterschiedlichkeit aufweisen müsste.

Dementsprechend hat der sachverständige Zeuge auf der letzten Seite des Gutachtens die Probe 03 nur als „nicht verkrustete" Oberfläche bezeichnet.

Weiter hat der sachverständige Zeuge geschildert, dass sich die elektronenmikroskopisch untersuchten Proben der Verkrustung wegen ihrer fotografisch festgehaltenen, sehr losen Struktur schon morphologisch nicht als Sinter darstellen. Soweit der Beklagte im Rahmen seiner Stellungnahme auf die durchgeführte Beweisaufnahme unter Zeugen- und Sachverständigenbeweis behauptet, die Ablagerungen seien entgegen der vom Zeugen geschilderten Beobachtung bei der vorgenommenen Probenahme nach eigener Erkenntnis nicht leicht abzuheben gewesen, so ist der Beweisantritt auf Vernehmung des Zeugen Dr. P. schon verspätet, §§ 525, 296 Abs. 2 ZPO. Der als Zeuge benannte Dr. P. war in allen Verhandlungs- und Beweisaufnahmeterminen anwesend und hätte, auch anlässlich der Vernehmung des Zeugen Dr. N., als präsenter Zeuge benannt werden können und müssen, denn das Gutachten $\mathrm{K} 6$ mit der bereits dort schriftlich festgehaltenen Beobachtung war schon mit der Klageschrift hereingereicht worden und dem Beklagten daher bekannt. Dessen Relevanz für die Entscheidung war spätestens bei der Anordnung der Vernehmung des sachverständigen Zeugen offensichtlich. Auch die angeblich abweichende Wahrnehmung des Dr. P. war dem Beklagten aus dem als Anlage K 12 zur Akte gereichten Schreiben vom 26. Juni 2009 gleichfalls bereits spätestens während des erstinstanzlichen Verfahrens bekannt geworden. Zum anderen ist der Einwand auch inhaltlich unbehelflich. Dem sachverständigen Zeugen, der Untersuchungen der hier beschriebenen Art berufsmäßig vornimmt und über langjährige Erfahrung verfügt, war das Ablösen der Proben aus der angeblichen Verkrustung jedenfalls problemlos möglich. Eine feste Verbindung zum Stein, wie sie zu erwarten gewesen wäre, wenn es sich bei der Verkrustung tatsächlich um „aus dem Stein wachsendes" Sintermaterial gehandelt hätte, war mithin nicht vorhanden. Ob auch dem Streitverkündeten ein Ablösen möglich war, ist bei dieser Sachlage ohne Bedeutung.

Der Zeuge hat die fotografisch festgehaltene Feststellung von - nicht sehr deutlich sichtbaren - Spuren des Einsatzes moderner Werkzeuge neben Bearbeitungsspuren, die wahrscheinlich nicht auf den Einsatz moderner Werkzeuge zurückzuführen seien, erläutert und hierin ein weiteres Indiz für die fehlende Authentizität des Objekts gesehen. Der Einwand des Beklagten, diese Ausführungen seien widersinnig, ist nicht nachvollziehbar. Insbesondere spricht der Einsatz von modernen Werkzeugen - wenn auch neben dem Einsatz herkömmlicher Werkzeuge - indiziell gegen ein Alter des Objekts von 1400 Jahren.

Der sachverständige Zeuge hat nachvollziehbar beschrieben, dass er bei der Untersuchung der Ablagerungen das Vorhandensein organischen Materials durch indirekte Nachweis- 
methode (Verdunstung und daraus folgender Gewichtsverlust bei entsprechender Erhitzung) festgestellt hat. Diesen Umstand hat der sachverständige Zeuge als Indiz für die Verwendung von Bindemitteln und damit als (weiteres) Indiz gegen die Qualifizierung der genommenen Proben als Sintermaterial gewertet. Dass das Vorhandensein organischen Materials grundsätzlich auch andere Ursachen haben kann, ist damit nicht in Abrede gestellt. Entsprechendes gilt im Hinblick auf das vom Zeugen festgestellte Fehlen von Eisenhydroxidspuren. Insoweit hat der sachverständige Zeuge Feststellungen aufgelistet, die den elektronenmikroskopisch gewonnenen Eindruck stützen und abrunden und dem elektronenmikroskopischen Befund nicht widersprechen, ohne für sich alleine schon einen bestimmten eindeutigen Schluss zuzulassen.

Der Einholung des beklagtenseits angebotenen Sachverständigengutachtens über tonige Böden bedarf es daher nicht.

Dass der sachverständige Zeuge keine chemische Analyse des angeblichen Sintermaterials vorgenommen hat, sondern das Material mittels Differenzialthermoanalyse untersucht hat, ergibt sich aus dem Gutachten K 6 und den mündlichen Erläuterungen des sachverständigen Zeugen.

Das Fehlen eines eindeutigen Analyseergebnisses, mit dem das Vorhandensein einer bestimmten Bindesubstanz nachgewiesen wäre, ist notwendige Folge der Untersuchungsmethode und Vorgehensweise des Zeugen. Die Einholung eines weiteren Gutachtens ist hierdurch aber nicht veranlasst. Schon die durchgeführten Untersuchungen und dabei gemachten Feststellungen des sachverständigen Zeugen in ihrer Gesamtschau sprechen deutlich für das Vorliegen einer neuzeitlichen Fälschung. Nimmt man darüber hinaus die Ausführungen der Sachverständigen Dr. R. in den Blick, so gibt es keinen vernünftigen Zweifel mehr daran, dass das streitgegenständliche Objekt eine neuzeitliche Fälschung darstellt.

2.2. Dieser Umstand stellt sich in rechtlicher Hinsicht als Sachmangel im Sinne der hier anwendbaren BGB-Vorschriften zum Kaufrecht dar.

(1) Auf das Vertragsverhältnis findet gemäß der Rechtswahlklausel in Ziff. $9 \mathrm{c}$ ) der Versteigerungsbedingungen („Es gilt ausschließlich deutsches Recht.") gemäß Art. 29 Abs. 3, 27 Abs. 1 EGBGB das deutsche materielle Recht Anwendung.

(2) Die Kaufsache ist aus den unter Ziff. 1 festgestellten Gründen mit einem Sachmangel behaftet, $\S 434$ Abs. 1 Satz 2 Nr. 2 BGB, denn ihr fehlt das Alter, das sie zu einem Kunstgegenstand und Sammelobjekt erheben würde (vgl. zur Rechtslage vor Inkrafttreten des Schuldrechtsmodernisierungsgesetzes: BGH, Urteil vom 15. Januar 1975 - VII ZR 80/73, BGHZ 63, 369). Damit fehlt ihr die zentrale Sacheigenschaft, welche unter Berücksichtigung der Verkehrskreise, an die sich das Angebot der ausdrücklich als Kunstauktion bezeichneten Vermarktungsaktion richtet, die objektive Eignung der Kaufsache zur gewöhnlichen Verwendung begründet. Ob außerdem aus der Katalogbeschreibung als einer dem Beklagten zurechenbaren, öffentlichen Äußerung - trotz des oben zitierten AGB-mäßigen Ausschlusses in Ziff. 2 b) der Versteigerungsbedingungen - eine Beschaffenheitsvereinbarung nach $\S 434$ Abs. 1 Satz 3 BGB folgt, kann dahinstehen. Schon objektiv durfte der am Erwerb interessierte Kunstsammler, an den sich das Angebot der [...]-Kunstauktion richtet, erwarten, dass es sich bei der angebotenen Skulptur nicht um eine neuzeitliche Nachahmung eines Ausgrabungsfundes, sondern um ein echtes Sammlerstück handelt, dessen Beschädigungen und Anhaftungen auf einem entsprechenden Alterungsprozess und nicht auf in Täuschungsabsicht durchgeführten Manipulationen beruhen.

2.3. Da der Mangel objektiv nicht behebbar ist, war der Kläger gemäß §§ 437 Nr. 2, 440, 323, 326 Abs. 5, 275 Abs. 1 BGB ohne Nachfristsetzung zum Rücktritt berechtigt. Ziff. 7 a) der als allgemeine Geschäftsbedingungen formulierten Versteigerungsbedingungen steht dem nicht entgegen, denn diese Klausel ist zum einen überraschend im Sinne von § 305c BGB und daher nicht Vertragsbestandteil geworden und zum anderen wegen unangemessener Benachteiligung der Vertragspartei des Verwenders gemäß § 307 BGB unwirksam.

(1) Allgemeine Geschäftsbedingungen sind nach ihrem objektiven Inhalt und typischen Sinn einheitlich so auszulegen, wie sie von verständigen und redlichen Vertragspartnern unter Abwägung der Interessen der regelmäßig beteiligten Verkehrskreise verstanden werden, wobei die Verständnismöglichkeiten des durchschnittlichen Vertragspartners zugrunde zu legen sind. Zweifel bei der Auslegung gehen nach § 305c Abs. 2 BGB zulasten des Verwenders. Außer Betracht bleiben nur solche Verständnismöglichkeiten, die zwar theoretisch denkbar, praktisch aber fernliegend und nicht ernstlich in Betracht zu ziehen sind (BGH, Urteil vom 21. April 2009 - XI ZR 78/08, BGHZ 180, 257 Rn. 11).

Nach diesem Maßstab der kundenfeindlichsten Auslegung besagt die Klausel, dass dem Käufer keinerlei Gewährleistungsansprüche gegen den Versteigerer wegen Sachmängeln zustehen, auch dann nicht, wenn der Käufer innerhalb der in Ziff. 7 a) Satz 2 der Bedingungen gesetzten Jahresfrist das Vorliegen eines Sachmangels dem Verkäufer nachweist. Diese Regelung erstreckt sich mangels Bereichsausnahme auch auf den Fall, dass der Käufer gegenüber dem Verkäufer den Nachweis der Fälschung der Kaufsache erbringt. Selbst dann, wenn der Verkäufer auf Kosten des Käufers mit Beweismaterial über das Vorliegen einer Fälschung versorgt und hierdurch in die Lage versetzt wird, unter Verwendung der Beweismittel seinerseits den Einlieferer auf Haftung in Anspruch zu nehmen, soll der Käufer keinen Anspruch gegen den Verkäufer haben, weder einen Anspruch auf Abtretung der dem Verkäufer im Rechtsverhältnis zum Einlieferer zustehenden Rechte noch einen Anspruch auf Auskehr einer - möglicherweise unter Verwendung der käuferseitig eingeholten Erkundungen und zur Verfügung gestellten Beweismittel durchgesetzten - Ersatzleistung des Einlieferers. 
Ein Anspruch auf Abtretung der dem Verkäufer zustehenden Gewährleistungsrechte gegen den Einlieferer ist nämlich nach dem Wortlaut der Klausel ebenso nicht gegeben wie ein Anspruch auf Auskehr durchgesetzter Ersatzleistungen. Vielmehr soll nach dieser Klausel der Verkäufer dem Käufer gegenüber lediglich verpflichtet sein, im eigenen Namen und für eigene Rechnung die nachgewiesenen und daher begründeten Mängelrügen geltend zu machen. Eine Verpflichtung, die Rechtsverfolgung zugunsten des Käufers durchzuführen, ist damit nicht gegeben.

Gemäß diesem kundenfeindlichsten Verständnis der Klausel verhält sich der Beklagte auch vorliegend. Diese Auslegung fällt daher nicht in die Kategorie der nicht zu berücksichtigenden, fernliegenden und nicht ernsthaft in Betracht zu ziehenden Verständnismöglichkeiten.

(2) Eine solche Regelung ist überraschend im Sinne von $\S 305$ c BGB.

Sie ist, wie ein Vergleich mit den vom Beklagten als Anlagen BE 2-BE 5 vorgelegten Versteigerungsbedingungen anderer Auktionshäuser zeigt, ungewöhnlich. Bei den dort verwendeten Versteigerungsbedingungen werden die Gewährleistungsausschlussklauseln durchgängig ergänzt durch die Einräumung von im Einzelnen unterschiedlich ausgestalteten Ansprüchen des Ersteigerers auf Partizipation an der Schadloshaltung des Versteigerers in dessen Verhältnis zum Einlieferer.

Ziff. 4 der Versteigerungsbedingungen des Auktionshauses L. bestimmt:

„L. verpflichtet sich [...] bei Abweichungen von den Katalogangaben, welche den Wert oder die Tauglichkeit aufheben oder nicht unerheblich mindern, und welche innerhalb eines Jahres nach Übergabe in begründeter Weise vorgetragen werden, seine Rechte gegenüber dem Einlieferer gerichtlich geltend zu machen. [...] Im Falle einer erfolgreichen Inanspruchnahme des Einlieferers erstattet L. dem Erwerber ausschließlich den gesamten Kaufpreis. Darüber hinaus verpflichtet sich L. für die Dauer von zwei Jahren bei erwiesener Unechtheit zur Rückgabe der vollständigen Kommission, wenn der Gegenstand in unverändertem Zustand zurückgegeben wird. Im Übrigen ist eine Haftung wegen Mängeln ausgeschlossen."

Beim Auktionshaus N. lautet die entsprechende Bestimmung in Ziff. 1. c):

„Der Versteigerer verpflichtet sich bei Sachmängeln, die innerhalb von 12 Monaten nach Zuschlag geltend gemacht wurden, seine Ansprüche gegenüber dem Einlieferer geltend zu machen, soweit der Käufer die Rechnung des Versteigerers vollständig bezahlt hat. Zur Geltendmachung eines Sachmangels ist die Vorlage des Gutachtens eines anerkannten Sachverständigen, welches den Mangel nachweist, auf Kosten des Käufers erforderlich. Im Falle erfolgreicher Inanspruchnahme des
Einlieferers erstattet der Versteigerer dem Käufer ausschließlich den Zuschlagspreis Zug um Zug gegen Rückgabe des Gegenstandes. Der Käufer bleibt zur Entrichtung des Aufgeldes als Dienstleistungsentgelt verpflichtet. Im Übrigen ist eine Haftung des Versteigerers wegen Sachmängeln ausgeschlossen."

Das Auktionshaus H. R. hat die Gewährleistung wie folgt geregelt (Ziff.3.):

„Der Versteigerer haftet nicht für Mängel, soweit er die ihm obliegende Sorgfaltspflicht bei der Beschreibung der versteigerten Gegenstände erfüllt hat. Er verpflichtet sich jedoch, wegen rechtzeitig vorgetragener begründeter Mängelrügen innerhalb der Verjährungsfrist seine Gewährleistungsansprüche gegenüber dem Einlieferer geltend zu machen. Im Falle erfolgreicher Inanspruchnahme des Einlieferers stellt der Versteigerer den Erwerber schadlos."

Beim Versteigerer E. (V. Kunstauktionen KG) lauten die entsprechenden Klauseln Ziff. 2.4 und 2.5:

"2.4 Eine Haftung von V. wegen etwaiger Mängel wird ausdrücklich ausgeschlossen, [...].

2.5 Weist der Käufer jedoch innerhalb eines Jahres nach Übergabe des Kunstwerkes nach, dass Katalogangaben über die Urheberschaft des Kunstwerkes unrichtig sind und nicht mit der anerkannten Meinung der Experten am Tag der Drucklegung übereinstimmten, verpflichtet sich $\mathrm{V}$. unabhängig von Ziffer 2.4, seine Rechte gegenüber dem Einlieferer geltend zu machen. Im Falle der erfolgreichen Inanspruchnahme des Einlieferers erstattet V. dem Erwerber ausschließlich den gesamten Kaufpreis. Darüber hinaus verpflichtet sich V. für die Dauer von zwei Jahren bei erwiesener Unechtheit zur Rückgabe der vollständigen Kommission. [...]."

Diese Zitate erhellen die objektive Ungewöhnlichkeit der vom Beklagten verwendeten Versteigerungsbedingungen. Mit einer solchen Klausel musste der Käufer auch nicht rechnen, denn es liegt auf der Hand, dass der Versteigerer bei Vorliegen nachweisbarer Sachmängel Gewährleistungsansprüche gegenüber dem Einlieferer geltend machen kann, während der Nachteil aus dem Vorhandensein des Sachmangels den Ersteigerer trifft mit der Folge, dass ohne Schaffung eines Ausgleiches der Versteigerer auf Kosten seines Vertragspartners, des Ersteigerers, einen Vorteil aus der Mangelhaftigkeit der Kaufsache ziehen könnte, während er nach dem Leitbild der gesetzlichen Gewährleistungsvorschriften im Falle der Mangelhaftigkeit der Kaufsache auch ohne eigenes Verschulden haftet. Der Klausel wohnt wegen dieser krassen Abweichung vom dispositiven Recht und wegen ihrer Abweichung von den bei anderen Auktionshäusern üblichen kundenfreundlicheren Bestimmungen ein Überrumpelungs- und Übertölpelungseffekt inne, weshalb diese Klausel nicht Vertragsbestandteil geworden ist, $\S 305 \mathrm{C}$ Abs. 1 BGB. 
(3) Die vom Beklagten verwendete Klausel ist daneben auch wegen unangemessener Benachteiligung des Kunden unwirksam, § 307 Abs. 1 Satz 1 BGB. Unangemessen ist die Benachteiligung dann, wenn der Verwender durch einseitige Vertragsgestaltung missbräuchlich eigene Interessen auf Kosten seines Vertragspartners durchzusetzen versucht, ohne von vornherein auch dessen Belange hinreichend zu berücksichtigen und ihm einen angemessenen Ausgleich zuzugestehen (BGH, Urteile 03. November 1999 - VIII ZR 269/98, BGHZ 143, 104 Rn. 31; vom 01. Februar 2005 - X ZR 10/04, NJW 2005, 1774 Rn. 21; vom 17. September 2009 - III ZR 207/08, NJW 2010, 57 Rn. 18). Die Unangemessenheit ist zu verneinen, wenn die Benachteiligung des Vertragspartners durch höherrangige oder zumindest gleichwertige Interessen des AGB-Verwenders gerechtfertigt ist.

Nach diesen Leitlinien ist die vom Beklagten verwendete Klausel unwirksam. Zwar hat der Bundesgerichtshof in der Entscheidung vom 13. Februar 1980 - VII ZR 26/79, WM 1980, 529, die Wirksamkeit einer AGB-mäßigen Freizeichnung des Auktionators bei Fälschungen bestätigt unter der Voraussetzung, dass der Auktionator seine Sorgfaltspflicht im Rahmen der Prüfung des eingelieferten Versteigerungsgutes erfüllt (so bereits vor Inkrafttreten des AGBG a. F.: BGH, Urteil vom 15. Januar 1975 - VIII ZR 80/73, BGHZ 63, 369). Angesichts des Umstandes, dass der Versteigerer nicht als Eigentümer, sondern nur als Kommissionär tätig werde, der den Versteigerungserlös abzüglich des Auktionsgeldes an den Einlieferer abführt, sowie des im Kunsthandel typischerweise erheblichen Risikos hinsichtlich der Echtheit des Kommissionsgutes und der Herkunftsangaben des Einlieferers werde der Gewährleistungsausschluss von den berechtigten Interessen des Auktionators getragen. Einen angemessenen Ausgleich des hierdurch bedingten Rechtsnachteils erhalte der Ersteigerer durch die Möglichkeit, das Versteigerungsgut bereits während einer angemessenen Frist vor dem Versteigerungstermin zu besichtigen, die Echtheit zu prüfen und Auskünfte einzuholen. Allerdings dürfe sich der Versteigerer dann nicht auf den wirksam vereinbarten Gewährleistungsausschluss berufen, wenn er die ihm obliegenden Sorgfaltspflichten bei der Hereinnahme des Versteigerungsgutes nicht beachtet hat. Dabei liege die Beweislast für die Wahrung der im Verkehr erforderlichen Beweislast beim Auktionator. In der dortigen Entscheidung hat es der Bundesgerichtshof dahinstehen lassen, ob der Auktionator dann, wenn er sich auf den formularmäßigen Haftungsausschluss berufen will, dem Ersteigerer die ihm gegen den Einlieferer zustehenden Ansprüche abtreten muss (Rn. 34 der genannten Entscheidung).

Seit dieser Entscheidung aus dem Jahr 1980 hat sich bei der Bewertung und Gewichtung der Verbraucherrechte und des Verbraucherschutzes ein Wandel eingestellt. Die dortigen Erwägungen überzeugen nicht mehr. Zwar treffen die Darlegungen des Bundesgerichtshofs zu den beiderseitigen Interessen der Vertragspartner einer in Kommission durchgeführten Versteigerung von Kunstgegenständen weiterhin zu. Ein angemessener Ausgleich der widerstreitenden Interessen und in diesem Zusammenhang eine angemessene Berücksichtigung der - auch vom Bundesgerichtshof anerkannten - Interessen des Ersteigerers erfordert aber zumindest einen Anspruch des Ersteigerers auf Teilhabe an den Rechten, die dem Versteigerer in seinem Verhältnis zum Einlieferer zustehen, insbesondere dann, wenn der Sachmangel in dem Umstand einer Fälschung liegt. Dabei sind - wie die Versteigerungsbedingungen anderer Auktionshäuser zeigen - unterschiedliche Gestaltungsmöglichkeiten denkbar, bei denen die berechtigten Interessen des Auktionators umfassende Berücksichtigung finden, zugleich aber auch die Interessen des Ersteigerers einen wirksameren Schutz erfahren als nach der hergebrachten Rechtslage. Diese hat zur Konsequenz, dass der Ersteigerer trotz geführten Nachweises einer Fälschung dann keinen Anspruch gegen den Auktionator geltend machen kann, wenn das Nichterkennen der Fälschung diesem nicht als sorgfaltspflichtwidrig vorzuwerfen ist, was angesichts der stetigen Verbesserung der Fälschungstechniken in einer Vielzahl der Fälle eintreten kann, andererseits aber der Auktionator - sogar unter Verwendung der Erkenntnisse aus dem kostenmäßig zum Nachteil des Ersteigerers gehenden Verfahrens - seinerseits erfolgreich den Einlieferer in Anspruch nehmen kann. Damit wird das Fälschungsrisiko einseitig dem Ersteigerer auferlegt, ohne diesem einen angemessenen Ausgleich zu gewähren, denn eine stärkere Berücksichtigung der Interessen des Ersteigerers ist - wie gezeigt - ohne weiteres und ohne jede Schmälerung der Interessen des Versteigerers möglich. Dies beurteilen auch andere Auktionshäuser offensichtlich nicht anders. Auch der Beklagte ist gehalten, nicht vor allem seine Interessen zu betonen und den Ersteigerer auf oftmals unzulängliche Untersuchungsmöglichkeiten am Ausstellungsstück und im Übrigen auf das Vertrauen in die Sorgfalt des Versteigerers bei der Prüfung des Auktionsgutes zu verweisen. Indem Ziff. 7 a) der Versteigerungsbedingungen des Beklagten keinen Anspruch des einen Sachmangel, insbesondere eine Fälschung, nachweisenden Ersteigerers auf Teilhabe an den dem Versteigerer im Verhältnis zum Einlieferer zustehenden Rechte vorsieht, erweist sich der klauselmäßige Gewährleistungsausschluss als gegen Treu und Glauben verstoßende, einseitige Benachteiligung des Käufers und ist daher unwirksam.

2.4. Gemäß den eingangs unter Gliederungspunkt 2. genannten Vorschriften kann der Kläger daher vom Beklagten Rückgewähr der erbrachten Kaufpreiszahlung in Höhe von 20.295,00 € Zug um Zug gegen Rückgewähr der empfangenen Leistung, nämlich Eigentum und Besitz an der streitgegenständlichen Buddha-Skulptur, verlangen.

Ersatz von Zinsen auf die zurückzugewährende Kaufpreiszahlung schuldet der Beklagte als Verzugsschaden gemäß § 286 Abs. 1 BGB ab dem Ablauf der mit vorprozessualem Schreiben vom 17. August 2010 [...] auf den 03. September 2010 gesetzten Frist.

Die seinerseits geschuldete Leistung hat der Kläger mit dem auf eine Zug-um-Zug-Verurteilung gerichteten Klageantrag in einer den Annahmeverzug begründeten Weise dem Beklagten, der die Statue an dem Ort, an dem sie sich vertragsgemäß 
befindet, mithin am Wohnort des Klägers abzuholen hat (BGH, Urteil vom 09. März 1983 - VIII ZR 11/82, NJW 1983, 1479 Rn. 15), mündlich angeboten, §§ 293, 295 Satz 1 BGB. Antragsgemäß war daher der Annahmeverzug des Beklagten festzustellen.

3. Der Kläger hat daneben einen Anspruch auf Ersatz der für das vorprozessual eingeholte Privatgutachten getätigten Aufwendungen von 1.339,51 $€$ [...], jedoch keinen Anspruch auf Ersatz des auf 2.255,00 $€$ bezifferten Währungsschadens, der infolge der seit Kaufpreiszahlung eingetretenen Aufwertung der schweizerischen Währung CHF im Verhältnis zum EUR als derjenigen Währung, in der der Kläger den Kaufpreis entrichtet hat, eingetreten ist.

Das klägerische Begehren ist insoweit nicht auf im Rückabwicklungsverhältnis nach §§ 346, 347 BGB berücksichtigungsfähige Positionen gerichtet, sondern auf Ersatz eines weitergehenden Vermögensschadens. Dieser ist nicht im begehrten Umfang vom Beklagten zu ersetzen.

(1) Grundsätzlich kann nach der dispositiven kaufvertraglichen Regelung bei vorliegendem Verschulden des Verkäufers neben dem Rücktritt auch eine Schadensersatzforderung geltend gemacht werden, $\S ₫ 437$ Nr. 3, 440, 281 BGB.

Der vom Beklagten angebotene Entlastungsbeweis war jedoch nicht einzuholen, denn die Verpflichtung zur Leistung von Schadensersatz für Vermögensschäden wurde ausweislich Ziff. 7b) der Versteigerungsbedingungen vertraglich ausgeschlossen. Diese AGB-mäßige Vertragsbestimmung ist wirksam. Sie hält sich in den durch $\S 309$ (Nr. 7) BGB gezogenen Grenzen. Die unter Gliederungspunkt 2. erörterten Bedenken gegen die Wirksamkeit des umfassenden Gewährleistungsausschlusses gemäß Ziff. 7 1) der Versteigerungsbedingungen jedenfalls bei erwiesener Fälschung der Kaufsache gelten nicht auch hinsichtlich des Ausschlusses der Schadensersatzpflicht für Vermögensschäden. Insoweit kann nicht festgestellt werden, dass dieser Ausschluss die Interessen des Auktionators unter Hintanstellung der Interessen des Ersteigerers unangemessen überbetone. Vielmehr erscheint diese Bestimmung unter Berücksichtigung der typischen Risikolage im Kunsthandel sowie der Erlösverteilung beim Kommissionsgeschäft als sachgerechte Risikozuweisung der den Kaufpreis übersteigenden etwaigen Vermögensschäden an den Ersteigerer und daher als unbedenklich.

Die in Ziff. 7 a) einerseits und Ziff. 7 b) andererseits getroffenen Regelungen betreffen zwar denselben Sachkomplex, nämlich die kaufvertragliche Gewährleistung, sind aber sprachlich und inhaltlich klar voneinander abtrennbar. Ziff. 7 der Versteigerungsbedingungen stellt sich daher als teilbare Klausel dar, deren unbedenklicher Teil (lit. b) - ohne Verstoß gegen das Verbot der geltungserhaltenden Reduktion - wirksam bleibt $(\mathrm{BGH}$, Urteil vom 25. Januar 2006 - VII ZR 3/05, NJW 2006, 1059 Rn. 22).
(2) Die vorprozessual gemachten Aufwendungen für den Privatsachverständigen sind vorliegend jedoch als "Kosten des Rechtsstreits" nach § 91 Abs. 1 ZPO erstattungspflichtig und ausnahmsweise mit Blick auf die Verweigerungshaltung des Beklagten bereits mit der Hauptsache einklagbar, denn auf das Kostenfestsetzungsverfahren als das grundsätzlich einfachere Verfahren ist der Kläger nicht zu verweisen. Eine Ersatzpflicht hinsichtlich der Kosten für ein vorprozessual eingeholtes Parteigutachten besteht nach $\S 91$ Abs. 1 ZPO dann, wenn eine ausreichende Klagegrundlage nur durch einen Sachverständigen beschafft werden konnte und das Gutachten zur Rechtsverfolgung nicht nur erforderlich, sondern auch prozessbezogen in Auftrag gegeben war (BGH, Beschluss vom 17. Dezember 2002 VI ZB 56/02, MDR 2003, 413). Diese Voraussetzungen sind erfüllt. Der Kläger hat das Gutachten aufgrund von Bedenken gegen die Echtheit der Statue in Auftrag gegeben, um auf der Basis einer sachverständigen Aussage seine Rechte gegen den Beklagten - auch im Prozesswege - zu verfolgen. Hierzu wurde er zudem durch die vom Beklagten verwendete Bestimmung in Ziff. 7 a) der Versteigerungsbedingungen angehalten. Das Gutachten wurde zudem im Prozess vorgelegt und hat dort im Rahmen der Beweisaufnahme Verwendung gefunden.

Zinsen auf den auszugleichenden Betrag schuldet der Beklagte erst ab Rechtshängigkeit, § 291 BGB, weil er insoweit ausweislich des Schreibens vom 17. August 2010 [...] nicht bereits zu einem früheren Zeitpunkt in Verzug gesetzt worden ist.

(3) Eine sonstige Anspruchsgrundlage für den begehrten Ersatz des Währungsschadens besteht hingegen nicht. Auf der Grundlage der den Streitgegenstand bestimmenden tatsächlichen Begründung des geltend gemachten Währungsschadens kann Ersatz eines - auf der Basis abweichender Stichtage und Bezugsdaten zu berechnenden - Währungsschadens auch nicht anstelle der geltend gemachten Zinsen auf den Hauptsachebetrag nach $\S 288$ Abs. 4 oder $\S 346$ Abs. 4 BGB zugesprochen werden, § 308 ZPO.

Insoweit verbleibt es daher bei der erstinstanzlich ausgesprochen Klageabweisung.

4. Nebenentscheidungen: Der Kostenausspruch beruht auf § 92 Abs.2 ZPO, der Ausspruch zur vorläufigen Vollstreckbarkeit auf §§ $708 \mathrm{Nr}$. 10, 711 ZPO.

Die Revision war mit Blick auf die unter Gliederungspunkt 2. bezeichnete Rechtsprechung des Bundesgerichtshofes zur Wirksamkeit eines klauselmäßigen Gewährleistungsausschlusses im Kunstauktionsgewerbe gemäß $§ 543$ Abs. 2 Nr. 2 ZPO zuzulassen.

Der Streitwert entspricht dem mit der Berufung weiterverfolgten Hauptsachebetrag. (Entscheidung von der Redaktion bearbeitet.) 